\title{
GENERALIZED LINEAR MODELS IN ACTUARIAL WORK
}

\author{
By S. Haberman and A. E. Renshaw \\ (A synopsis of a paper presented to the Society on 2 February 1988)
}

THE use of classical linear models in actuarial work is not new. Such models have become an established part of the description of claim frequency rates and average claim costs in motor insurance - as evidenced by a number of papers, including Johnson and Hey ${ }^{(1)}$, Grimes ${ }^{(2)}$, Bennett ${ }^{(3)}$, Baxter et al. ${ }^{(4)}$ and Coutts ${ }^{(5)}$.

However, the use of generalized linear models in actuarial work is relatively new. Thus, McCullagh and Nelder ${ }^{(6)}$ give a number of examples of the fitting of generalized linear models to different types of data. One of these relates to data from Baxter $e t$ al..$^{(4)}$ on the average claim costs in a motor insurance portfolio (originally modelled by Baxter et al. using a weighted least-squares approach). We made a small step in the direction of using generalized linear models in life insurance when we modelled the variation of lapse rates with age at entry, duration of policy, type of policy and insurance company ${ }^{(7)}$. Some of these models are described further in this paper.

The purpose of the paper is to show that generalized linear models have a wide area of application in actuarial work and are not confined merely to models for motor insurance premiums. This purpose is fulfilled by demonstrating three separate practical applications in actuarial work:

(i) fitting of loss distributions in non-life insurance (Section 3);

(ii) representing the variation in force of mortality in life insurance underwriting (Section 4); and

(iii) representing the variation in lapse rates with policy characteristics in life insurance (Section 5).

Each of these applications involves different types of data and a different type of model.

Section 3 is concerned with the fitting of skewed loss distributions. It shows how to adapt the GLIM package to fit certain types of distribution which are not immediately available within the package. Three examples are the Pareto, Burr and Weibull distributions. It is a trivial matter to fit other loss distributions such as the log normal, gamma and log gamma to uncomplicated data.

The applications discussed in Section 4 are particularly novel. The GLIM-based approach outlined here could pave the way for a completely new, scientifically sound approach to life insurance underwriting. It offers a more dynamic means of model building than has hitherto been attempted in this field. The models allow the effects of individual factors and their interactions on excess mortality may be assessed. We would highlight the meager assumptions on which the models are based, the comparative ease with which they can be fitted and compared using GLIM and the appealing connection which these models have with the traditional actuarial approach using standard mortality ratios. 
Section 5 indicates how Generalized Linear Models may be applied to a wide range of problems where an actuarial rate or probability, or a one-year risk premium can be represented as a function of a set of significant rating factors. Section 5 deals specifically with lapse rates but earlier authors have also examined the components of motor insurance risk premiums (i.e. claim frequency and average claim cost). There is considerable scope for extending these ideas into other areas (e.g. group life risk premiums or marine insurance risk premiums).

Both Sections 4 and 5 deal with generalized linear models of large complex data sets. Modelling such large complex data sets may be viewed as a balancing act between model complexity and the need to encapsulate the salient underlying features present in the data. The simpler the model, the simpler the interpretation of the underlying data generating mechanism. Modelling does not necessarily have a unique solution, but a model may be deemed adequate only if it achieves this goal.

One way of assessing the adequacy is through a thorough graphical analysis of model residuals which, ideally, should be 'pattern free'. Additionally, what might be termed 'fine tuning' may then be attempted, and its effects formally assessed.

\section{REFERENCES}

(1) Johnson, P. D. and Hey G. B. (1971) Statistical Studies in Motor Insurance. J.I.A., $97,199$.

(2) Grimes T. (1971) Claim Frequency Analysis in Motor Insurance. J.S.S., 19, 147.

(3) Bennett M. (1978) Models in Motor Insurance. J.S.S., 22, 134.

(4) Couts S. M. (1984) Motor Insurance Rating: An Actuarial Approach. J.I.A., 111, 87.

(5) Baxter L. A., Coutts S. M. and Ross G. A. F. (1980) Applications of Linear Models in Motor Insurance. Proceedings of the 21st International Congress of Actuaries, 2, 11.

(6) McCullagh P. and Nelder J. R. (1989) 2nd ed. Generalised Linear Models. Chapman \& Hall.

(7) Renshaw A. E. and Haberman S. (1986) Statistical Analysis of Life Assurance Lapses. J.I.A., 133, 459. 